\title{
The 4.1 Protein Coracle Mediates Subunit-Selective Anchoring of Drosophila Glutamate Receptors to the Postsynaptic Actin Cytoskeleton
}

\author{
Kaiyun Chen, ${ }^{1}$ Carlos Merino, ${ }^{2}$ Stephan J. Sigrist, ${ }^{2}$ and David E. Featherstone ${ }^{1}$ \\ ${ }^{1}$ Department of Biological Sciences, University of Illinois at Chicago, Chicago, Illinois 60607, and ${ }^{2}$ European Neuroscience Institute Göttingen, Max-Planck- \\ Society, 37073 Göttingen, Germany
}

\begin{abstract}
Glutamatergic Drosophila neuromuscular junctions contain two spatially, biophysically, and pharmacologically distinct subtypes of postsynaptic glutamate receptor (GluR). These receptor subtypes appear to be molecularly identical except that A receptors contain the subunit GluRIIA (but not GluRIIB), and B receptors contain the subunit GluRIIB (but not GluRIIA). A- and B-type receptors are coexpressed in the same cells, in which they form homotypic clusters. During development, A- and B-type receptors can be differentially regulated. The mechanisms that allow differential segregation and regulation of A- and B-type receptors are unknown. Presumably, Aand B-type receptors are differentially anchored to the membrane cytoskeleton, but essentially nothing is known about how Drosophila glutamate receptors are localized or anchored. We identified coracle, a homolog of mammalian brain 4.1 proteins, in yeast two-hybrid and genetic screens for proteins that interact with and localize Drosophila glutamate receptors. Coracle interacts with the $\mathrm{C}$ terminus of GluRIIA but not GluRIIB. To test whether coracle is required for glutamate receptor localization, we immunocytochemically and electrophysiologically examined receptors in coracle mutants. In coracle mutants, synaptic A-type receptors are lost, but there is no detectable change in B-type receptor function or localization. Pharmacological disruption of postsynaptic actin phenocopies the coracle mutants, suggesting that A-type receptors are anchored to the actin cytoskeleton via coracle, whereas B-type receptors are anchored at the synapse by another (yet unknown) mechanism.
\end{abstract}

Key words: actin; Drosophila; postsynaptic; genetics; glutamate receptor; 4.1

\section{Introduction}

Most excitatory synaptic transmission in mammalian brains is mediated by ionotropic glutamate receptors (GluRs). The processes controlling glutamate receptor expression, localization, and stabilization are subjects of intense study (Monaghan and Wenthold, 1997; Bolton et al., 2000; De La Rue and Henley, 2002). However, these processes remain incompletely understood.

The Drosophila genome encodes 21 putative ionotropic glutamate receptor subunits, including homologs of mammalian NMDA, AMPA, kainate, and $\delta$ receptor subunits (Sprengel et al., 2001). Five subunits, called GluRIIA, GluRIIB, GluRIIC (also known as GluRIII), GluRIID, and GluRIIE, are postsynaptically expressed in the glutamatergic neuromuscular junction (NMJ) (Qin et al., 2005). Genetic and physiological analyses suggest that these five subunits combine to form two distinct glutamate receptor subtypes: A receptors and B receptors (Marrus et al., 2004;

Received April 19, 2005; revised June 2, 2005; accepted June 6, 2005.

This work was supported by a National Institutes of Health/National Institute of Neurological Disorders and Stroke R01 (D.E.F.) and a grant from the Alexander Humbolt Foundation (C.M.). Thanks to Qi (Betty) Sheng for technical assistance.

Correspondence should be addressed to David Featherstone, Department of Biological Sciences, University of Illinois at Chicago, 840 West Taylor Street, M/C 067, Chicago, IL 60607. E-mail: def@uic.edu.

DOI:10.1523/JNEUROSCI.1527-05.2005

Copyright $\odot 2005$ Society for Neuroscience $\quad$ 0270-6474/05/256667-09\$15.00/0
Chen and Featherstone, 2005; Featherstone et al., 2005; Qin et al., 2005). A and B receptors are thought to be molecularly identical except for one subunit: A-type receptors contain the subunit GluRIIA but not GluRIIB. B-type receptors contain the subunit GluRIIB but not GluRIIA. In addition to either GluRIIA or GluRIIB, all postsynaptic fly NMJ glutamate receptors contain GluRIIC, GluRIID, and GluRIIE. A- and B-type receptors have functional differences (DiAntonio et al., 1999) and are spatially segregated as homotypic clusters (Marrus et al., 2004; Chen and Featherstone, 2005). Presumably, A and B receptors differentially associate with membrane-associated proteins and/or cytoskeletal components, but this has never been tested because the molecular mechanisms that localize and anchor Drosophila glutamate receptors are almost completely unknown. To elucidate these mechanisms, we are conducting separate yeast two-hybrid and genetic screens for proteins that interact with and localize NMJ glutamate receptors. These screens independently identified the Drosophila 4.1 protein coracle.

Mammalian glutamate receptor subunits have been shown to interact with 4.1 proteins, and this interaction is thought to be important for trafficking and/or localization of receptors to synaptic membrane (Shen et al., 2000; Baines et al., 2001; Coleman et al., 2003). However, this requirement has yet to be demonstrated in vivo, and it is not clear which mammalian receptor subtypes rely on this mechanism. 
4.1 proteins were first identified in erythrocytes, in which they link transmembrane proteins to the spectrin-actin cytoskeleton. Actin is abundant postsynaptically and has been proposed to anchor glutamate receptors (Shirao and Sekino, 2001; Aarts and Tymianski, 2004). Pharmacological disruption of actin in cultured mammalian neurons disrupts NMDA receptor currents (Rosenmund and Westbrook, 1993), but it is unclear whether actin is required for localization or stabilization of other glutamate receptor subtypes. A requirement for spectrin in mammalian glutamate receptor localization has never been shown. In Drosophila, elimination of $\alpha$ or $\beta$ spectrin causes mislocalization of several synaptic proteins but no electrophysiologically detectable change in postsynaptic glutamate receptors (Featherstone et al., 2001). The question of whether or not postsynaptic actin is required for Drosophila NMJ function has never been addressed.

Here, we show that Drosophila A-type receptors, but not B-type receptors, are anchored to the postsynaptic actin cytoskeleton via subunit-dependent interactions with the 4.1 protein coracle.

\section{Materials and Methods}

Genetics. Control genotype was $w[1118]$ or nonhomozygous mutant siblings of the appropriate genotype. No statistically significant difference in any measurement was observed between $w[1118]$, any other control genotype used in this study, or the widely used wild-type control Oregon $R$. GluRIIA[AD9] specifically removes GluRIIA and has been described previously (Petersen et al., 1997; Marrus et al., 2004). cora [k08713] mutants were obtained from the Bloomington Stock Center (Indiana University, Bloomington, IN). $D f(2 R) B S C 26$ is a deletion of the coracle gene derived from imprecise excision of cora[k08713] (Parks et al., 2004). The null mutant cora[5] was a gift from Dr. Richard Fehon (University of Chicago, Chicago, IL) and has been described previously (Ward et al., 1998). Homozygous mutant embryos were identified through the use of an appropriate balancer chromosome expressing green fluorescent protein.

Immunocytochemistry. Embryos and larvae were dissected and stained as described previously (Featherstone et al., 2002; Chen and Featherstone, 2005). When antibodies against either GluRIIA or GluRIIB were used, preparations were fixed for $30 \mathrm{~min}$ in Bouin's fixative. Otherwise, fixations were $30 \mathrm{~min}$ in $4 \%$ paraformaldehyde. Monoclonal mouse hybridoma cell antibodies against GluRIIA (8B4D2, used at 1:100) were obtained from the Developmental Studies Hybridoma Bank (University of Iowa, Iowa City, IA). Rabbit polyclonal GluRIIB antibodies (Marrus et al., 2004) were gifts from Dr. Aaron DiAntonio (Washington University, St. Louis, MO) and used at 1:2000. The mouse polyclonal coracle antibody A0784 was obtained from the Center for Biomedical Inventions (CBI) Antibody Core Facility (University of Texas Southwestern Medical Center, Dallas, TX) and used at 1:500. The mouse monoclonal coracle antibody 9C (Fehon et al., 1994) was a gift from Dr. Richard Fehon. All primary antibodies were visualized using fluorescently conjugated [fluorescein, rhodamine, or cyanine 5 (Cy5)] secondary antibodies (Jackson ImmunoResearch, West Grove, PA) generated against the appropriate species (mouse or rabbit) and viewed using an Olympus Optical (Tokyo, Japan) FV-500 laser-scanning confocal microscope. Presynaptic terminals were visualized using fluorescently conjugated anti-HRP antibodies (Jackson ImmunoResearch) directly conjugated to FITC, tetramethylrhodamine isothiocyanate, or Cy5. Actin was visualized using rhodamine-conjugated phalloidin (Molecular Probes, Eugene, OR). All measurements were performed on the ventral longitudinal muscle 6/7 NMJ.

Electrophysiology. All electrophysiology was performed on ventral longitudinal muscle 6 . These whole-cell patch-clamp measurements were performed as described previously (Featherstone et al., 2001, 2002; Chen and Featherstone, 2005). Briefly, temporally and morphologically staged embryos or first-instar larvae were manually dissected, and then muscles were treated for 60-90 s with $1 \mathrm{mg} / \mathrm{ml}$ collagenase type IV (SigmaAldrich, St. Louis, MO) to aid seal formation. Muscle 6 was whole-cell patch clamped $(-60 \mathrm{mV})$ in standard Drosophila saline using standard patch-clamp techniques. Data were acquired and subsequently analyzed using an Axopatch 1D amplifier and pClamp 9 (Axon Instruments, Union City, CA).

Yeast two-hybrid experiments. Two-hybrid experiments were performed using the Clontech (Cambridge, UK) Matchmaker system. In the original screen, a Drosophila embryo Matchmaker cDNA Library (Clontech) was screened using GluRIIA C-terminal region. Positive clones isolated from this screening were sequenced using the standard Clontech primers. The sequence corresponding to one clone contained the last 926 base pairs of coracle-RA cDNA; this clone was named "Coracle C-term." To analyze the interaction between Coracle C-term and the glutamate receptors, sequential transformations in $\mathrm{AH} 109$ yeast strain were performed using GluRIIA, GluRIIB, GluRIIC, GluRIID, and GluRIIE C terminal as bait and pACT2-Coracle C-term as prey. The protein interaction was analyzed in media lacking histidine, adenine, leucine, and tryptophane (-HALT medium), as well as in medium lacking tryptophane and leucine containing B-galactosidase. In all of the experiments, pGBKT7-p53 and pGADT7-T antigen cotransformation was used as positive control. The negative controls included were the cotransformation of pACT2-Coracle C-term with the empty plasmid pGBKT7 or with pGBKT7-laminin and the cotransformation of pGADT7 empty plasmid with the plasmids containing the different glutamate receptor C-terms. Glutamate receptor C-terminal regions were obtained by PCR and clone in pGBKT7 (Clontech) between EcoR1 and XhoI sites. Only in the case of GluRIIE, the amplified fragment was cloned between SmaI and XhoI sites. The primers used to amplified the glutamate receptors were as follows: GluRIIA C-term, 5' -gagctcgagttcctgtggaacgtgcag-3', 5' -ctcgaattcctagctaaccgtcttgctgcg 3'; GluRIIB C-term, 5' -gagctcgagtttttgtggcacgtccag-3' 5' $^{\prime}$-ctcgaattcctacttttcaattcgcctggt- $3^{\prime}$; GluRIIC C-term, 5' -gagctcgagttttggtctacgtgcagc-3', $5^{\prime}$-ctcgaattcttagaccettgcctccttttc-3'; GluRIID C-term, 5' -gcgacccgggatgttggtgctactttgtctacaagaaag- $3^{\prime}$, 5' -gctcctcgagttaatcctcaaccggcatattttcct- $3^{\prime}$; and GluRIIE C-term, 5'-gcgagaattcagctgggttttgttcgtaatgaagaaagc- $3^{\prime}, 5^{\prime}$-gctcctcgagctactgcgattcctgggccacagaag- $3^{\prime}$.

Pharmacology. Pharmacological disruption of postsynaptic actin was performed by adding $25 \mu \mathrm{M}$ latrunculin A or $25 \mu \mathrm{M}$ cofilin (Molecular Probes) to the solution in the patch pipette. For imaging, latrunculin was added to the dissected preparation for $10 \mathrm{~min}$; the preparation was then fixed. For control experiments (on either control or coracle mutant animals), the same period of time normally taken for drug treatment was allowed to elapse before electrophysiological recording or fixing of the preparation.

Statistics. Statistical significance in figures is represented as follows: ${ }^{\star} p<0.05 ;{ }^{* *} p<0.01 ;{ }^{* * *} p<0.001$. Unless otherwise specified, $n$ refers to the number of different animals examined, and all statistical comparisons were made using unpaired $t$ tests or (in the case of distributions) Kolmogorov-Smirnov tests. All error bars represent SEM.

\section{Results}

The localization and segregation of Drosophila NMJ A- and B-type receptors is likely to be mediated by interactions between specific receptor subunit intracellular regions and other proteins. In mammals, the intracellular $C$ termini of glutamate receptor subunits have been shown to be important for interactions with other postsynaptic density (PSD) proteins. To find proteins required for A-type receptor localization, we used the GluRIIA C terminus as bait in a yeast two-hybrid screen of a Drosophila embryonic cDNA library (Clontech). This screen recovered a C-terminal fragment of coracle. Coracle is thought to be the only 4.1 protein encoded by the Drosophila genome. However, this presupposes no subsequent discovery of any Drosophila 4.1 homologs after completion and reannotation of the fly genome. Mammalian genomes encode four major 4.1 proteins, known as $4.1 \mathrm{R}, 4.1 \mathrm{G}, 4.1 \mathrm{~N}$, and $4.1 \mathrm{~B} .4 .1 \mathrm{~N}$ is most abundant postsynaptically in hippocampal neurons (Shen et al., 2000), and 4.1R is most abundant in cerebellar granule cell PSDs (Ohara et al., 2000). 4.1R is also present in PSD extracts (Scott et al., 2001). 4.1G is primarily non-neuronal (Ohara et al., 2000). To confirm that 
A

ERM
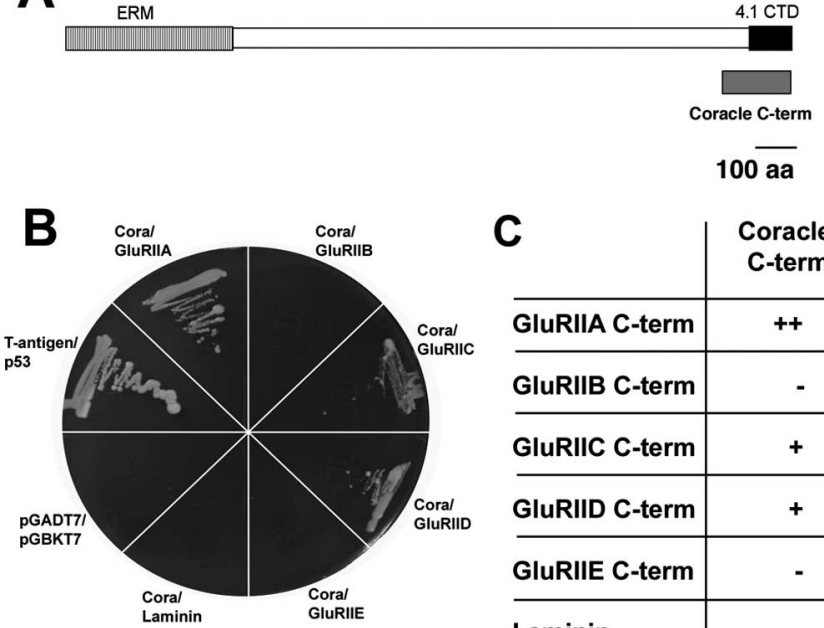

\begin{tabular}{l|c} 
C & $\begin{array}{c}\text { Coracle } \\
\text { C-term }\end{array}$ \\
\hline GluRIIA C-term & ++ \\
\hline GluRIIB C-term & - \\
\hline GluRIIC C-term & + \\
\hline GluRIID C-term & + \\
\hline GluRIIE C-term & - \\
\hline Laminin & -
\end{tabular}

Figure 1. Coracle 4.1 C-terminal domain (CTD) interacts with GluRIIA, GluRIIC, and GluRIID. $A$, Representation of Coracle RA protein showing the ezrin/radixin/moesin (ERM) domain and the 4.1 protein C-terminal domain. Coracle C-term corresponds to the last 91 amino acids (aa) of coracle. This clone was recovered in the screening of Drosophila embryonic CDNA library (Clontech) using GluRIIA C-term as bait. B, Analysis of the interaction of Coracle C-term with the C-term of the different glutamate receptors. Yeast cotransformed with Coracle and the different glutamate receptor C-term growth in - HALT medium. T-antigen/p53 represents positive control and pGADT7/pGBKT7, and coracle C-term/lamin represents the negative controls. C, Summary of the interaction between Coracle C-term and glutamate receptors. ++ represents a strong interaction, + represents a weak interaction, and - represents no interaction.

coracle represents the sole fly homolog of all of these 4.1 subtypes, we performed searches with the basic local alignment search tool BLASTP using mouse 4.1R, 4.1G, $4.1 \mathrm{~N}$, and $4.1 \mathrm{~B}$ amino acid sequences using the most recently annotated version of the Drosophila genome (release 4.1) and confirmed that coracle is the closest fly homolog to all of these mammalian proteins.

To confirm the interaction between coracle and GluRIIA and determine whether coracle might interact with other receptor subunits, we tested for an interaction between the 91 amino acid $\mathrm{C}$ terminus of coracle (which contains the $4.1 \mathrm{C}$-terminal domain) (Fig. 1A) and the $\mathrm{C}$ termini of each of the five glutamate receptor subunits expressed in Drosophila embryonic/larval body wall muscle: GluRIIA, GluRIIB, GluRIIC, GluRIID, and GluRIIE. As shown in Figure $1 B$, we observed a strong direct interaction between coracle and GluRIIA, with weaker interactions between coracle and GluRIIC, and coracle and GluRIID. No interaction was observed between coracle and GluRIIB. These results are summarized in Figure $1 C$.

Independently, we identified coracle mutants in a forward genetic screen for P-element mutants showing disruptions in A-type receptor expression and localization visible by confocal microscopy. $P\{w[+m C]=l a c W\}$ cora $[k 08713]$ is a publicly available P-element insertion mutant generated by the ongoing Berkeley Drosophila Gene Disruption Project (Bellen et al., 2004). In these cora[k08713] mutants, a P-element is inserted into the second intron of the coracle gene (Fig. $2 A$ ). As we show below (Figs. $2 \mathrm{~B}, 3 \mathrm{H})$, several lines of evidence suggest that this P-element insertion specifically disrupts coracle expression: (1) homozygous cora[k08713] mutants show reduced coracle immunoreactivity; (2) cora[k08713] fails to complement the previously described null allele cora[5]; (3) cora[k08713] fails to complement the deficiency Df(2R)BSC26, which was generated by excision of cora $[k 08713]$ and completely removes the coracle gene (Parks et
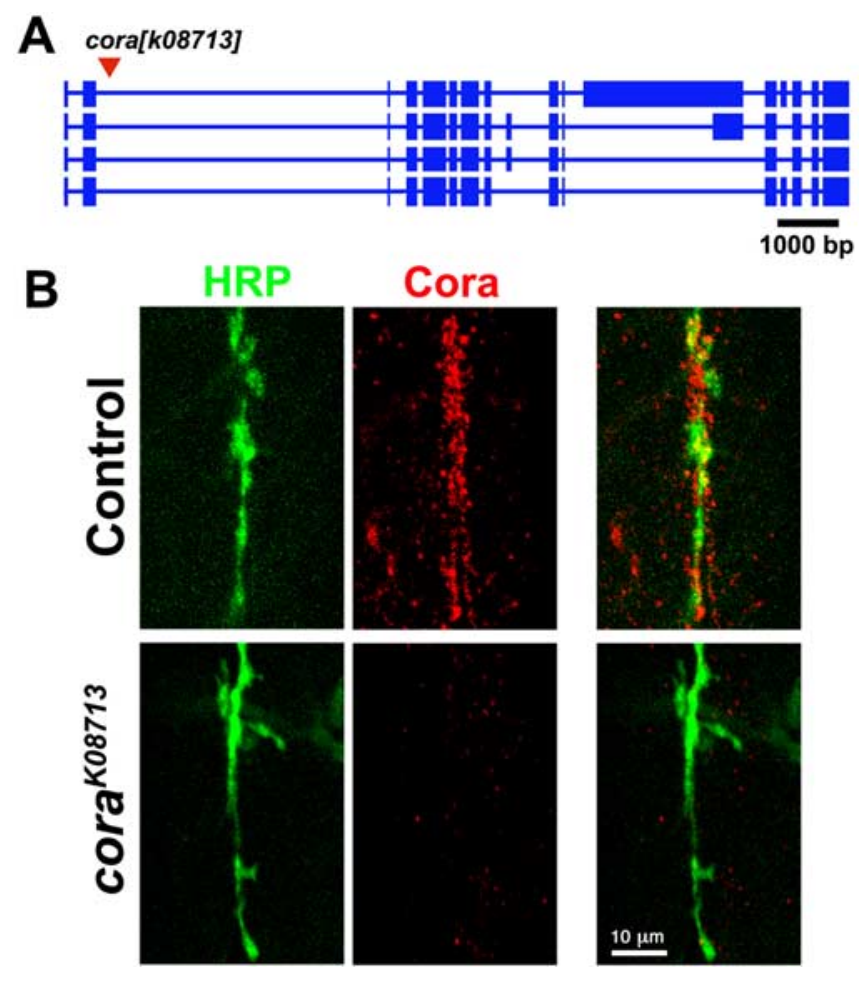

Figure 2. Coracle is present in Drosophila embryonic neuromuscular junctions and decreased in cora[k08713] mutants. $\boldsymbol{A}$, Diagram showing coracle gene structure and isoforms. Thick bars represent exons; thin bars represent introns. The insertion site of $P\left\{w[+m C]=\right.$ lacW\}cora[k08713], in the $5^{\prime}$ end of the second intron, is indicated by the red arrowhead. An insertion at this position is predicted to affect all isoforms equally. $\boldsymbol{B}$, Embryonic NMJs on ventral longitudinal muscles 6/7 stained using anti-HRP antibodies (which recognize neuronal membrane, green) and A0784 anti-coracle antibodies (red). As shown in the figure, coracle is distributed throughout the muscle membrane, but immunoreactivity is enriched postsynaptically. In homozygous cora[k08713] mutants, presynaptic morphology is normal but coracle immunoreactivity is greatly reduced. 9 C anti-coracle antibodies (Fehon et al., 1994) give virtually identical staining (data not shown).

al., 2004); and (4) homozygous cora [k08713] mutants phenocopy cora [5] homozygous mutants (Fig. 3H). Together, the genetic, immunocytochemical, and electrophysiological data (see below) show that homozygous cora $[k 08713]$ mutants behave genetically as zygotic null alleles. However, because there appears to be some maternal contribution, embryonic cora[k08713] mutants are probably most accurately functionally described as strong protein hypomorphs.

These yeast two-hybrid and genetic screen results suggest that coracle may be required for localization of A-type glutamate receptors at the Drosophila NMJ. If this is true, coracle must be present at the NMJ. However, the neuromuscular expression distribution of coracle has never been described. To determine whether coracle is at the NMJ, we examined coracle protein expression using two different antibodies. The first antibody was a previously undescribed mouse polyclonal antibody (A0784) generated by the University of Texas Southwestern Medical Center CBI Antibody Core against a recombinant peptide matching coracle amino acids 1185-1284. The second antibody (9C) has been described previously (Fehon et al., 1994). Both antibodies gave essentially identical expression. As reported previously, coracle is expressed in a variety of tissues. However, we were specifically interested in whether coracle was present in embryonic/L1 NMJs, for two reasons: (1) we are interested in determining whether coracle localizes/segregates embryonic/larval NMJ 
A

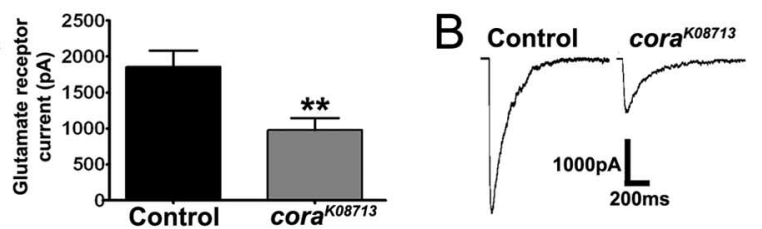

C Control
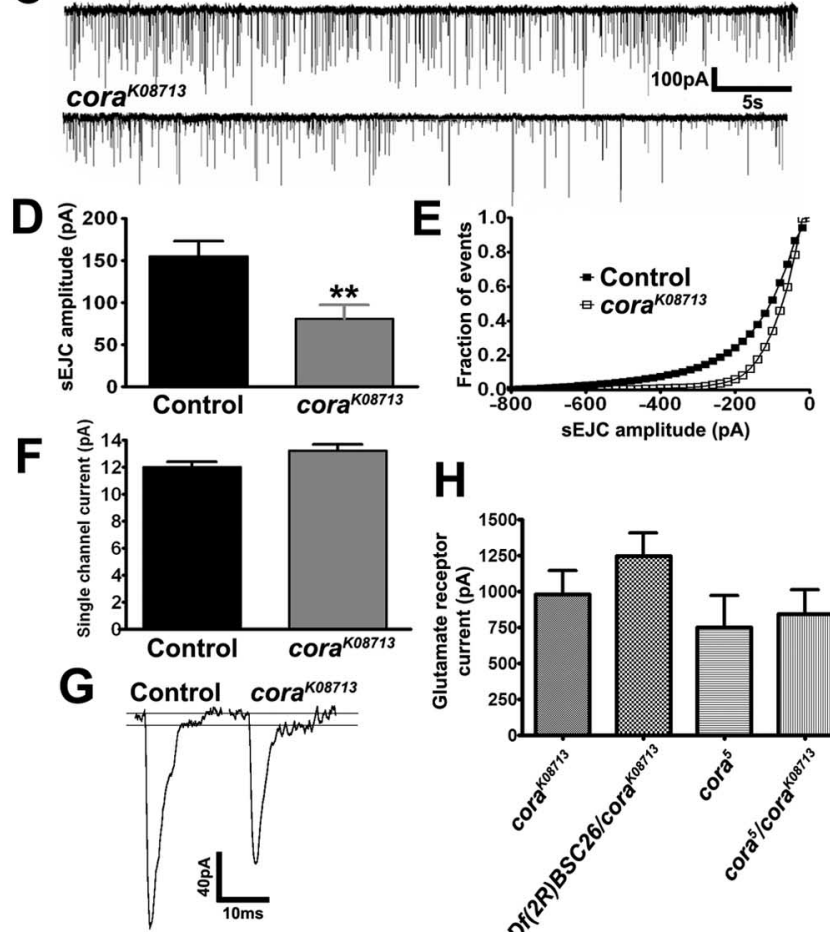

H

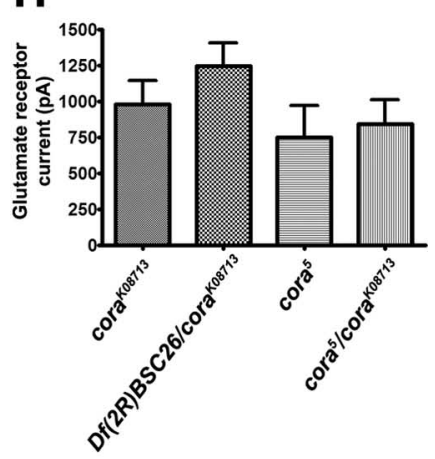

Figure 3. The number of functional postsynaptic glutamate receptors is reduced in coracle mutants. $\boldsymbol{A}$, The amplitude of glutamate-gated currents triggered by pressure ejection of $1 \mathrm{~mm}$ glutamate onto patch-clamped muscle 6 is reduced to approximately one-half normal in homozygous cora[k08713] mutants. Sample data from each genotype are shown in B. C, Sample continuous patch-clamp recordings from the muscle 6 NMJ. cora[08713] shows robust spontaneous synaptic transmission, as evidenced by the frequent sEJCs (downward deflections) over time. $\boldsymbol{D}$, The mean sEJC amplitude in cora[k08713] mutants is decreased to approximately one-half normal. $\boldsymbol{E}$, Cumulative frequency histogram of $s E J C$ amplitudes, showing that the largest sEJCs tend to be preferentially reduced in cora[k08713] mutants, consistent with other mutations that cause loss of postsynaptic glutamate receptors (Featherstone et al., 2005). $\boldsymbol{F}$, Single glutamate receptor current amplitudes are normal in cora [k08713] mutants. G, Single glutamate receptor current amplitudes are measured from delayed single receptor closings visible during the falling phase of some $s E J C s$. $\boldsymbol{H}$, The electrophysiologically detectable loss of glutamate receptors in cora[k08713] mutants is phenocopied in other previously described coracle null alleles (e.g., cora[5]) and trans-heterozygotes (e.g., Df(2R)BSC26/cora[k08713] and cora[5]/cora[k08713]).

glutamate receptors, and (2) because coracle mutants are embryonic/first-instar lethal, and therefore all experiments on coracle mutants have to be performed at this stage or earlier. In the embryonic neuromusculature, coracle immunoreactivity is distributed throughout body wall muscles but is particularly dense in the NMJ (Fig. 2B). This is consistent with the known role of coracle in other Drosophila cell junctions and with the fact that 4.1 proteins are found in mammalian glutamatergic PSDs. Coracle immunoreactivity is also present in third-instar NMJs, in which coracle appears prominent in NMJ-associated tissues that likely represent glia (supplemental data, available at www.jneurosci.org as supplemental material). In homozygous cora[k08713] mutants (see below), neuromuscular immunoreactivity is dramatically reduced, although not completely eliminated (Fig. 2B).
The presence of coracle in the NMJ, along with the interactions between coracle and glutamate receptor subunits, suggests that coracle might be important for localization of NMJ glutamate receptors. If this is true, then glutamate receptors should be lost from the NMJ in coracle mutants. The most direct way of measuring the number of functional glutamate receptors is by pressure ejection of glutamate. To test whether the number of functional NMJ glutamate receptors is reduced in coracle mutants, we pressure ejected $1 \mathrm{~mm}$ glutamate onto patch-clamped $(-60 \mathrm{mV})$ postsynaptic ventral longitudinal muscle 6 in control and coracle mutant embryos. The results are shown in Figure 3, $A$ and $B$. In control muscles, pressure ejection of glutamate triggers a current that is $1858 \pm 222 \mathrm{pA}(n=13$ animals $)$. In homozygous cora $[k 08713]$ mutants, the glutamate-gated current amplitude is reduced to approximately one-half of the normal level: $979 \pm 167$ $\mathrm{pA}(n=12 ; p=0.004)$.

Our pressure ejection results suggest that the total number of functional receptors in coracle mutants is reduced. However, pressure ejection activates all functional surface receptors and thus assays both synaptic and extrasynaptic receptors. This may underestimate a reduction in receptors localized specifically to synapses. Spontaneous excitatory junction currents (sEJCs), by definition, represent activation of synaptic receptors. To determine whether coracle mutants have a reduced number of functional glutamate receptors localized specifically to synapses, we measured sEJC amplitudes (Fig. 3C-E). In control animals, the average sEJC amplitude was $154 \pm 18 \mathrm{pA}(n=10)$. In homozygous cora [ $k 08713]$ mutants, sEJC amplitude was reduced to $80 \pm$ $16 \mathrm{pA}(n=9 ; p=0.008)$. The reduction in sEJC amplitude, to $\sim 50 \%$ normal, is very similar to the reduction in glutamate-gated current amplitude (Fig. $3 A, B$ ). This suggests that glutamate receptors in coracle mutants are not dispersed from the PSD throughout the membrane, in which they would be activated by pressure ejection of glutamate. sEJC frequency was reduced, but not significantly, in cora[k08713] mutants (control sEJC frequency, $12.6 \pm 1.1 \mathrm{~Hz}, n=10$; cora $[k 08713]$ frequency, $9.1 \pm 2.1$ $\mathrm{Hz}, n=9 ; p=0.17)$. sEJC decay time was significantly reduced in coracle mutants (control sEJC decay time, $3.35 \pm 0.06 \mathrm{~ms}, n=$ 10 ; cora $[k 08713]$ decay time, $1.66 \pm 0.02 \mathrm{~ms}, n=9 ; p<0.0001)$. This latter change is consistent with a specific loss of A-type receptors, which have increased single-channel open times compared with B-type receptors (DiAntonio et al., 1999) (see below).

It is important to note that reductions in SEJC amplitude and glutamate-gated current could be explained not only by loss of functional receptors but by a change in the biophysical properties of individual receptors such that average single-channel conductance is decreased. As described previously (Nishikawa and Kidokoro, 1995; Featherstone et al., 2000, 2002, 2005; Chen and Featherstone, 2005), the single-channel amplitude of synaptic glutamate receptors in embryonic Drosophila muscles are measurable from delayed receptor closings observable on some sEJCs (Fig. 2G). In both control and cora[k08713] mutants, the single glutamate receptor channel amplitude was similar (Fig. $2 F, G$ ). Specifically, control single receptor amplitudes were $12.0 \pm 0.4$ pA ( $n=90$ events from 10 animals). cora[k08713] mutant single receptor amplitudes were $13.2 \pm 0.4 \mathrm{pA}(n=57$ events from 9 animals). These data, in combination with the pressure ejection and sEJC measurements, suggest a loss of approximately one-half of all functional postsynaptic glutamate receptors in coracle mutants. In Figure $2 \mathrm{H}$, we show data confirming that this phenotype is shared by other coracle alleles.

To confirm the loss of postsynaptic glutamate receptors in coracle mutants, we performed immunocytochemical experi- 

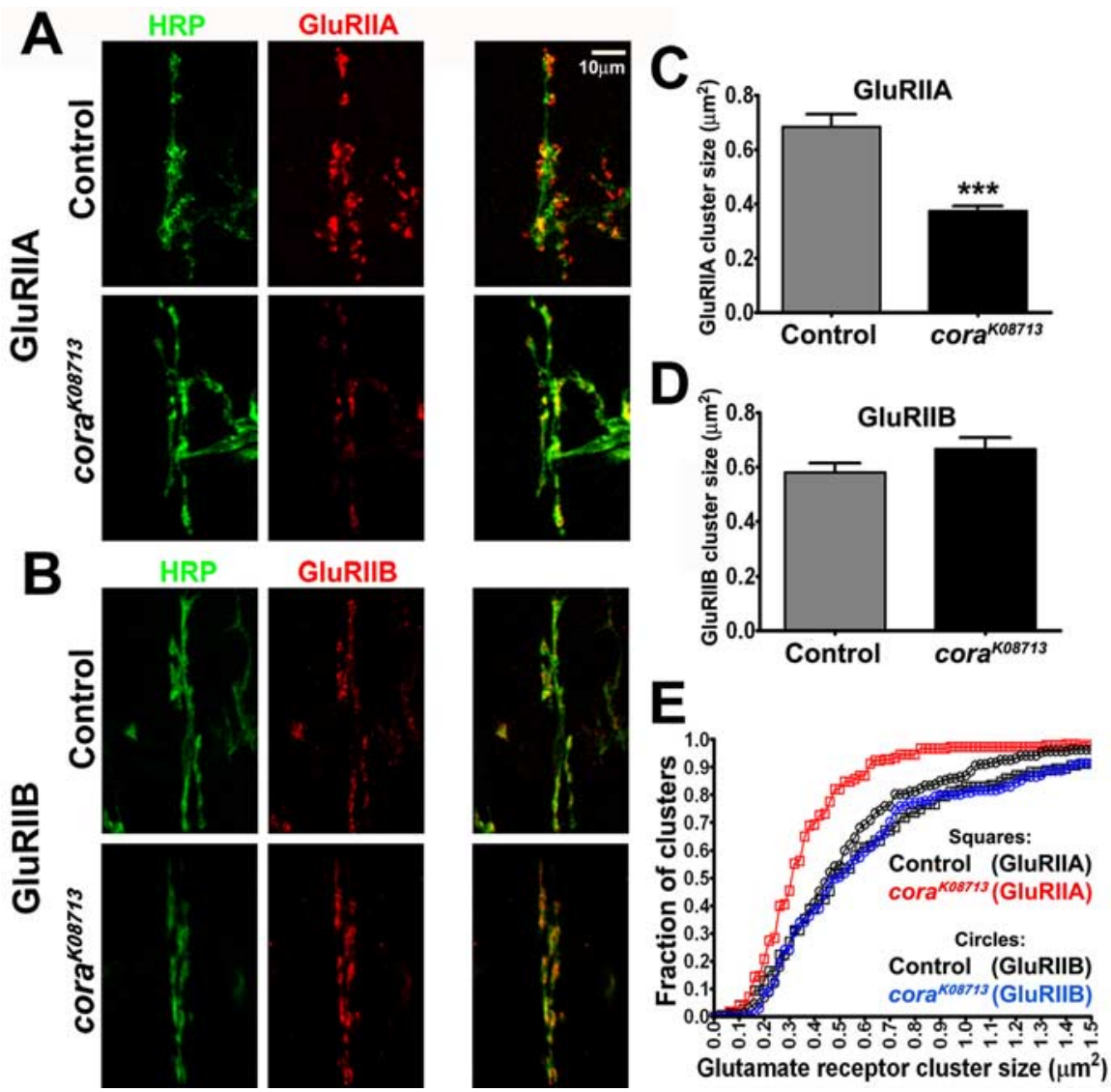

Figure 4. GluRIIA, but not GluRIII, is reduced in cora[k08713] mutants. A, GluRIIA immunoreactivity (red) in control embryonic NMJs appears as puncta, representing clusters of A-type glutamate receptors in PSD sopposite active zones (Marrus and DiAntonio, 2004; Chen and Featherstone, 2005) in presynaptic terminals (labeled with anti-HRP, green). In cora[k08713], GluRIIIA puncta are still present but are smaller and fewer in number. B, GluRIIB immunoreactivity (red), like GluRIIB immunoreactivity, is punctate. Each GluRIIB punctum represents a cluster of B-type glutamate receptors in a PSD opposite a presynaptic active zone (Marrus and DiAntonio, 2004; Chen and Featherstone, 2005). GluRIIB immunoreactivity in cora[k08713] mutant NMJs appears similar to that of controls. C, Quantification of GluRIIIA cluster size in control and cora[k08713] mutant embryonic NMJs, showing that mean A-type receptor cluster size in mutant NMJs is approximately one-half that of controls. In addition, there are fewer clusters (data not shown). D, Quantification of GluRIIB cluster size in control and cora[k08713] mutant embryonic NMJs, showing that mean $B$-type receptor cluster size in mutant NMJs is not significantly different from that of controls. $E$, Cumulative frequency histograms of cluster sizes, showing that the largest GluRIIA clusters tend to be preferentially reduced (and GluRIIB clusters unchanged) in cora[k08713] mutants.

ments on the same synapses examined electrophysiologically (embryonic muscle $6 \mathrm{NMJs)}$ ). A-type receptors were visualized using an antibody specific for the subunit GluRIIA. B-type receptors were visualized using an antibody specific for the subunit GluRIIB. As shown in Figure 4 and as described previously (Featherstone et al., 2002, 2005; Chen and Featherstone, 2005), clusters of A- and B-type glutamate receptors in control Drosophila embryonic muscles are detectable as immunoreactive puncta. To quantify changes in the number of postsynaptic glutamate receptors, we measured the area of individual puncta. Cluster size measurements (compared with fluorescence intensity measurements) avoid a requirement for fluorescence intensity calibration or problems associated with potential differences in background immunofluorescence between genotypes. Assuming constant receptor density, cluster size also has the advantage of being directly proportional to the number of clustered receptors. Indeed, immunoreactive cluster sizes correlate well with high-resolution patch-clamp electrophysiological measurements between genotypes (Featherstone et al., 2002; Chen and Featherstone, 2005) and throughout embryonic/larval NMJ development (D. E.
Featherstone, unpublished observations). In homozygous cora[k08713] mutants, the average cluster size of A-type glutamate receptors is reduced to approximately onehalf of normal (Fig. 4A, C,E) (control GluRIIA cluster size, $0.68 \pm 0.46 \mu \mathrm{m}^{2}, n=$ 170 clusters from 11 animals; cora $[k 08713]$ GluRIIA cluster size, $0.37 \pm 0.18 \mu \mathrm{m}^{2}, n=$ 183 clusters from 11 animals; $p<0.0001$ ). The cluster size of B-type glutamate receptors, however, was not reduced in coracle mutants compared with controls (Fig. $4 B, D, E$ ) (control GluRIIB cluster size, $0.58 \pm 0.03 \mu \mathrm{m}^{2}, n=192$ clusters from 9 animals; cora[k08713] GluRIIB cluster size, $0.66 \pm 0.04 \mu \mathrm{m}^{2}, n=141$ clusters from 9 animals; $p=0.12$ ). This suggests that coracle mutant NMJs contain fewer A-type receptors but that the number of B-type receptors is unchanged.

4.1 protein is thought to localize and anchor membrane proteins via interactions with the actin cytoskeleton, spectrin cytoskeleton, or both actin and spectrin simultaneously. $\alpha$ and $\beta$ spectrins are expressed in the Drosophila neuromusculature and abundant at embryonic/larval NMJs (Featherstone et al., 2001). However, we can rule out the possibility that coracle anchors NMJ glutamate receptors to the spectrin cytoskeleton because NMJ glutamate receptor function is normal in Drosophila $\alpha$ and $\beta$ spectrin mutants (Featherstone et al., 2001). It has never been tested whether actin plays a role in localization of Drosophila glutamate receptors.

If actin plays a role in localization or stabilization of postsynaptic glutamate receptors, then actin might be enriched postsynaptically. F-actin can be visualized using fluorescently conjugated actinbinding toxin phalloidin. A difficulty with visualizing postsynaptic actin in the Drosophila NMJ, however, is that actin is part of the contractile cytoskeleton and, therefore, actin is abundant throughout the muscle cell. As a result, phalloidin staining is very strong and widespread throughout the postsynaptic muscle cell. However, using careful confocal sectioning, we observed a previously undescribed phalloidinsensitive "halo" of postsynaptic actin surrounding presynaptic boutons in the Drosophila NMJ (Fig. 5A-E). This halo suggests that Drosophila NMJs contain a previously unrecognized specialized postsynaptic actin-based cytoskeleton. Drosophila embryonic/L1 NMJs do not contain postsynaptic protrusions comparable with spines. To test whether postsynaptic actin is required for localization of NMJ glutamate receptors, we needed to examine receptors after disruption of the postsynaptic actin cytoskeleton.

Actin is critical for many aspects of embryonic development; therefore, we cannot genetically eliminate actin and study the effect on NMJ function. Actin, however, can be acutely disrupted pharmacologically (Fig. 5F-I). To pharmacologically disrupt postsynaptic actin, we delivered $25 \mu \mathrm{M}$ of either latrunculin A (which inhibits actin polymerization by binding to G-actin) or 
cofilin (which depolymerizes actin filaments) directly to the postsynaptic muscle cell via a patch pipette and electrophysiologically measured the number of synaptic glutamate receptors. Drug delivery via postsynaptic patch pipette has the advantage of avoiding/minimizing disruption of presynaptic actin, which is known to play important roles in synaptic function (Doussau and Augustine, 2000; Kuromi and Kidokoro, 2003). After gaining wholecell access (and thus delivering the actin inhibitors), sEJC amplitudes decreased within minutes, suggesting that actin is critical for maintenance of synaptic receptor function and that this actin is highly dynamic (and thus dependent on free $\mathrm{G}$-actin pools sequestered by latrunculin). In control animals, mean sEJC amplitude decreased from $154 \pm 18 \mathrm{pA}(n=10)$ to $99 \pm 13 \mathrm{pA}(n=11 ; p=0.02)$ after postsynaptic delivery of latrunculin A (Fig. $5 F, G)$. In cora [k08713] mutants, latrunculin A had no effect (Fig. 5F,G) (cora[k08713] sEJC amplitude, $80 \pm 16$ $\mathrm{pA}, n=9$; cora[k08713] plus latrunculin A, $86 \pm 17 \mathrm{pA}, n=12 ; p=0.82$ ). Similar results were obtained using cofilin in the patch pipette (Fig. $5 H-I$ ). Control sEJC amplitudes were $154 \pm 18 \mathrm{pA}(n=10)$ but reduced to $100 \pm 11 \mathrm{pA}(n=8)$ after cofilin treatment $(p=0.03)$. In cora [ $k 08713]$ mutants, cofilin had no effect (cora[k08713] sEJC amplitude, $80 \pm 16$ pA, $n=9$; cora $[k 08713]$ plus cofilin, $92 \pm$ $19 \mathrm{pA}, n=6 ; p=0.64)$. These results suggest that dynamic filamentous actin is required for maintenance of postsynaptic function, but that this requirement is eliminated in coracle mutants.

If coracle anchors A-type receptors via interactions with the postsynaptic actin cytoskeleton, then disruption of actin should phenocopy coracle mutants. To test whether pharmacological disruption of actin specifically results in loss of GluRIIAcontaining A-type receptors, we performed immunocytochemical experiments similar to those described for Figure 4. The results of these experiments are shown in Figure 6. After $10 \mathrm{~min}$ treatment with $25 \mu \mathrm{M}$ latrunculin, the size of A-type receptor clusters in control animals was reduced from $0.64 \pm 0.08 \mu \mathrm{m}^{2}$ ( $n=49$ clusters from 5 animals) to $0.28 \pm 0.04 \mu \mathrm{m}^{2}(n=20$ clusters from 5 animals) (Fig. $6 A-C)(p=0.01)$. In cora $[k 08713]$ mutants, latrunculin A had no effect on GluRIIA cluster size (Fig. $6 D-F)\left(\right.$ cora[k08713] cluster size, $0.38 \pm 0.3 \mu \mathrm{m}^{2}, n=66$ clusters from 7 animals; cora $[k 08713]$ plus latrunculin A, $0.30 \pm 0.02$ $\mu \mathrm{m}^{2}, n=33$ clusters from 6 animals; $p=0.09$ ).

In contrast, the size of B-type receptor clusters was not affected by latrunculin A in either controls or cora[k08713] mutants (Fig. 6G-L). Control GluRIIB cluster size was $0.62 \pm 0.3$ $\mu \mathrm{m}^{2}$ ( $n=121$ clusters from 8 animals) without latrunculin A treatment and $0.56 \pm 0.02 \mu \mathrm{m}^{2}(n=159$ clusters in 9 animals $)$ after latrunculin A treatment (Fig. $6 G-I)(p=0.21)$. GluRIIB cluster sizes in cora $[k 08713]$ mutants were $0.67 \pm 0.02 \mu \mathrm{m}^{2}(n=$
214 clusters from 9 animals) without latrunculin A treatment and $0.62 \pm 0.02 \mu \mathrm{m}^{2}$ ( $n=232$ clusters from 10 animals) after latrunculin A treatment (Fig. $6 J-L)(p=0.12)$. Coracle immunoreactivity in third-instar NMJs was not obviously altered by latrunculin treatment (supplemental data, available at www.jneurosci. org as supplemental material).

If actin is required for localization and/or stabilization of only A-type receptors, then latrunculin A should have no effect in the absence of A-type receptors. A- and B-type glutamate receptors are differentiated by subunit composition; A-type receptors contain the subunit GluRIIA (but not GluRIIB), whereas B-type receptors contain the subunit GluRIIB (but not GluRIIA). Deletion of the gene encoding GluRIIA does not cause lethality but eliminates A-type receptors in the NMJ. GluRIIA[AD9] mutants contain a deletion that specifically removes the GluRIIA gene (Petersen et al., 1997; Marrus and DiAntonio, 2004). In homozygous GluRIIA[AD9] mutants, all A-type receptors are gone, but the remaining B-type receptor clusters are normal size $(0.56 \pm 0.2$ $\mu \mathrm{m}^{2}, n=120$ clusters from 8 animals). To test whether actin is required in the absence of A-type receptors, we electrophysiologically and immunocytochemically measured the number of NMJ glutamate receptors in GluRIIA[AD9] mutants, with and without latrunculin treatment. As shown in Figure 7, latrunculin A in GluRIIA[AD9] mutants had no effect on receptor cluster size 
A

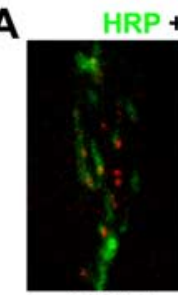

Control

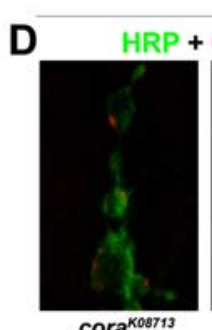

cora $^{\mathrm{Ko8713}}$

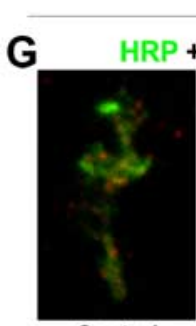

Control

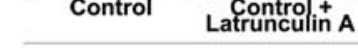

$\mathrm{J}$

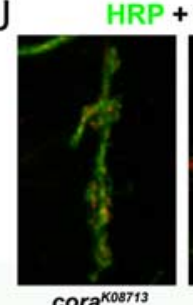

cora $^{\mathrm{Ko8713}}$

Latruncu
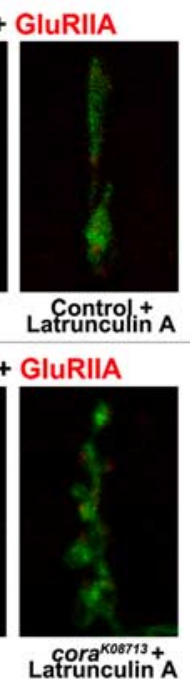

Latrunculin $^{\text {Cora }}$
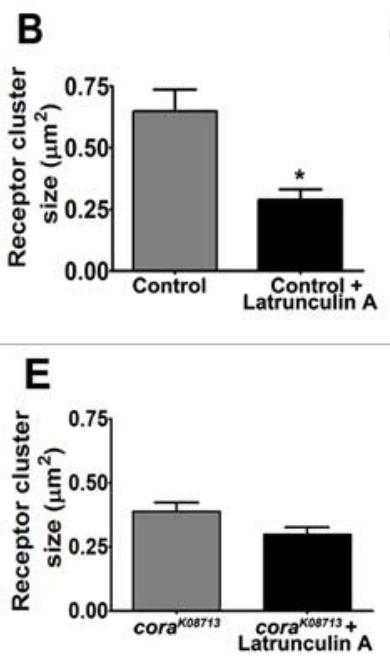

$\mathbf{F}$
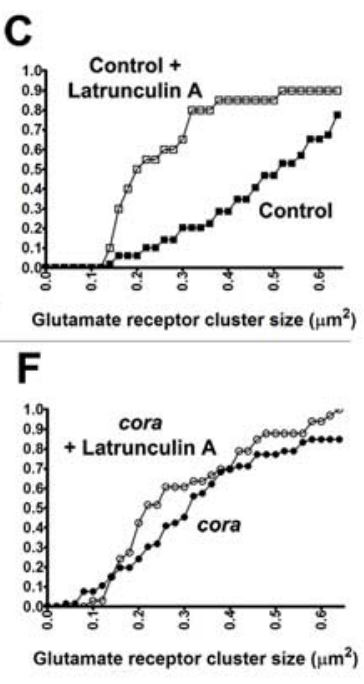

\section{I}
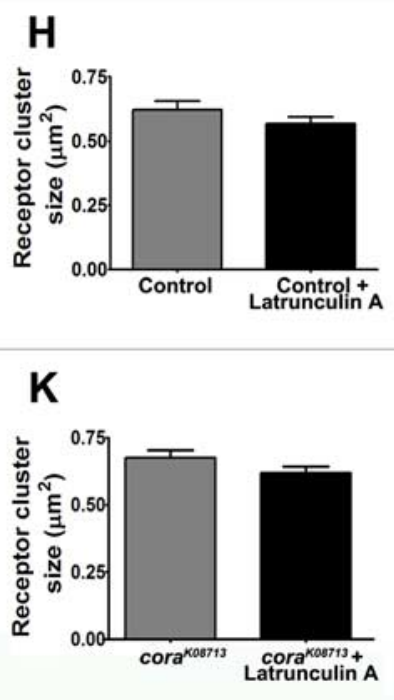
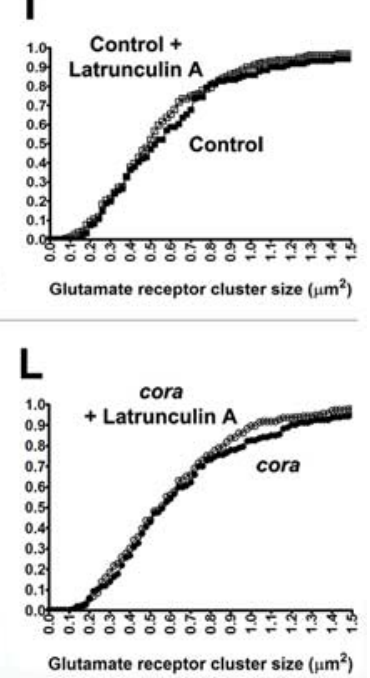

Figure 6. Pharmacological disruption of actin reduces GluRIIA, but not GluRIIB, in control animals but not coracle mutants. $\boldsymbol{A}$, As in Figure 4, GluRIIA immunoreactivity (red) normally appears as puncta opposite presynaptic terminals (green). In control NMJs, this immunoreactivity is reduced after treatment with latrunculin A. Specifically, A-type receptor cluster sizes get smaller, and the number of clusters is reduced. B, Quantification of GluRIIA cluster size in control NMJs, showing that average GluRIIA cluster size is reduced to approximately one-half normal after treatment with latrunculin A. C, Cumulative frequency histogram of GluRIIA cluster sizes in control NMJs, with and without latrunculin A treatment. D, As shown in Figure 4, GluRIIA immunoreactivity is reduced in coracle mutants. This immunoreactivity is not affected by treatment with latrunculin A. E, Quantification of GluRIIA cluster size in cora[k08713] mutant NMJs, showing no effect of latrunculin A. F, Cumulative frequency histogram of GluRIIA cluster sizes in coracle mutant NMJs, with and without latrunculin A treatment. G, As in Figure 4, GluRIIB immunoreactivity (red) normally appears as puncta opposite presynaptic terminals (green). GluRIIB immunoreactivity is not reduced by treatment with latrunculin A. $\boldsymbol{H}$, Quantification of GluRIIB cluster size in control NMJs, with and without treatment by latrunculin A, showing that latrunculin A has no effect on GluRIIB cluster size. I, Cumulative frequency histogram of GluRIIB cluster sizes in control NMJs that were (control plus latrunculin A) or were not (control) treated with latrunculin A. J, GluRIIB immunoreactivity is not reduced in coracle mutants compared with controls, and GluRIIB immunoreactivity in coracle mutants is not altered by treatment with latrunculin A. $\boldsymbol{K}$, Quantification of GluRIIB cluster size in coracle mutants showing no effect of latrunculin A. $L$, Cumulative frequency histogram of GluRIIB cluster sizes in coracle mutants, with and without latrunculin A treatment.

(Fig. $7 B, C$ ) or sEJC amplitude (Fig. $7 D, E$ ) (receptor cluster size in GluRIIA[AD9] mutants exposed to control saline, $0.56 \pm 0.2$ $\mu \mathrm{m}^{2}, n=120$ clusters from 8 animals; receptor cluster size in GluRIIA[AD9] mutants after treatment with latrunculin A, $0.60 \pm 0.2 \mu \mathrm{m}^{2}, n=13$ clusters from 8 animals; $p=0.21$ ). Similarly, postsynaptic latrunculin had no effect on sEJC amplitude in GluRIIA[AD9] mutants. Mean sEJC amplitude in GluRI$I A[A D 9]$ mutants (without latrunculin treatment) was $81 \pm 10$ $\mathrm{pA}(n=10)$, approximately one-half the amplitude of wild-type animals, consistent with complete and specific loss of A-type receptors. Latrunculin treatment did not change mean sEJC amplitude in GluRIIA[AD9] mutants (sEJC amplitude in GluRIIA[AD9] plus latrunculin, $76 \pm 10 \mathrm{pA}, n=8 ; p=0.77)$.

\section{Discussion}

We have described, for the first time, the presence of postsynaptic coracle and actin in the glutamatergic Drosophila NMJ and tested for the first time whether they are required for formation and maintenance of postsynaptic glutamate receptor clusters in vivo. We found that coracle and actin are required for proper clustering and stabilization of A-type glutamate receptors (which contain the subunits GluRIIA, GluRIIC, GluRIID, and GluRIIE) but not B-type glutamate receptors (which contain the subunits GluRIIB, GluRIIC, GluRIID, and GluRIIE). Our proposed model for stabilization of A-type receptors in the Drosophila NMJ is depicted in Figure 8. This mechanism for glutamate receptor anchoring is likely to be conserved; 4.1 protein and actin have also been implicated in mammalian glutamate receptor localization (Rosenmund and Westbrook, 1993; Shen et al., 2000; Baines et al., 2001; Shirao and Sekino, 2001; Coleman et al., 2003; Aarts and Tymianski, 2004).

Part of the evidence supporting our model is derived from yeast two-hybrid experiments. Yeast two-hybrid experiments showed that the $\mathrm{C}$ terminus of GluRIIA, but not GluRIIB, interacts strongly with the $4.1 \mathrm{C}$-terminal domain of coracle. However, the C termini of GluRIIC and GluRIID also interacted with coracle, although less strongly. Because GluRIIC and GluRIID are shared by A- and B-type receptors (Marrus et al., 2004; Featherstone et al., 2005; Qin et al., 2005), interactions between coracle and GluRIIC and GluRIID must be relatively unimportant for segregation of A- and B-type receptors. This is an important result; it suggests that protein interactions do not always accurately predict mechanisms by which receptors are anchored.

The mechanism by which B-type receptors are anchored is not yet known. Recent work (Chen and Featherstone, 2005) showed that the Drosophila PSD-95/ synapse-associated protein (SAP) 97/SAP102 homolog discs large (DLG) is required for stabilization of embryonic B-type receptors but not A-type receptors. However, the mechanism by which DLG functions is unclear because yeast two-hybrid experiments suggest no interaction between DLG and GluRIIA, GluRIIB, or GluRIIC (S. J. Sigrist, unpublished observations; U. Thomas, personal communication; as discussed by Chen and Featherstone, 2005). 

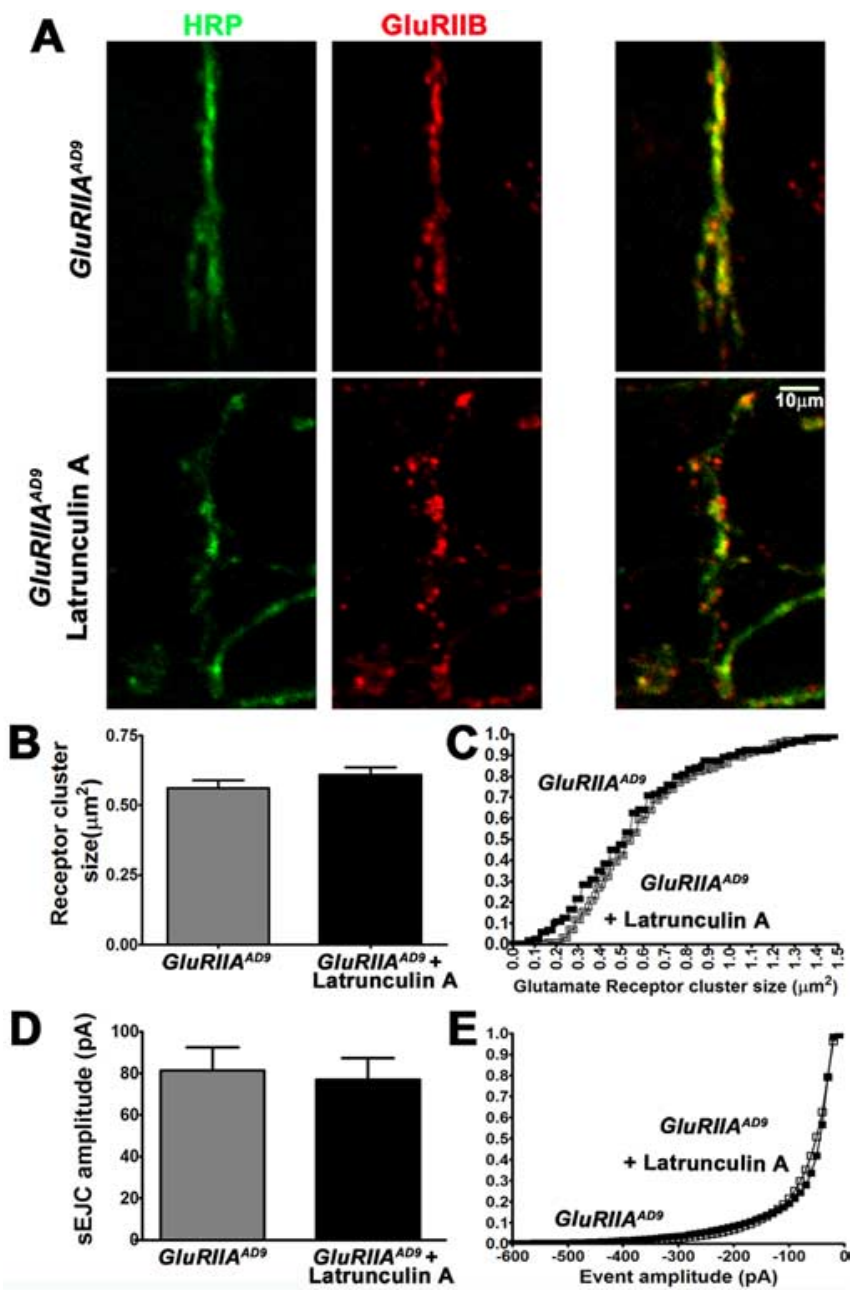

Figure 7. Pharmacological disruption of actin has no effect in the absence of GluRIIIA. $A$, In GluRIIA[AD9] mutants, which do not express GluRlIA, presynaptic morphology (anti-HRP, green) is normal but only B-type receptors (visualized using anti-GluRIIB, red) are present in the NMJ. In the absence of A-type glutamate receptors, treatment with latrunculin A causes no apparent change in the receptor immunoreactivity. $\boldsymbol{B}$, Quantification of glutamate receptor cluster size in GluRIIA[AD9] mutants, with or without latrunculin $A$ treatment. Latrunculin does not affect GluRIIA[AD9] mutant NMJ receptor cluster size. C, Cumulative frequency histogram of receptor cluster sizes in GluRIIA[AD9] mutants with (GluRIIA[AD9] plus latrunculin A) and without (GluRIIA[AD9]) treatment with latrunculin A. D, Latrunculin A delivered to the cytoplasm of the postsynaptic cell via patch pipette does not affect sEJC amplitude in GIURIIA[AD9] mutants. $\boldsymbol{E}$, Cumulative frequency histogram of SEJC amplitudes in GluRIIA[AD9] mutants, with and without latrunculin A treatment.

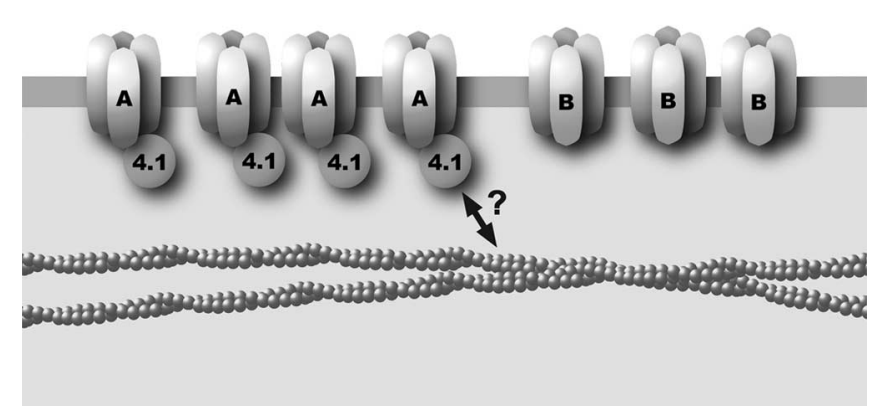

Figure 8. Proposed model for stabilization of A-type receptors in the Drosophila NMJ. Our data support a direct interaction between A-type receptors and the 4.1 protein coracle, but it remains unknown whether coracle interacts directly or indirectly with the actin cytoskeleton. $A$, A-type receptors; B, B-type receptors.
We emphasize that there is still no answer to the following question: What localizes glutamate receptor proteins in Drosophila? Drosophila NMJ glutamate receptors are clustered in small subsections of the postsynaptic membrane opposite presynaptic active zones. Both coracle and actin, conversely, are broadly distributed postsynaptically. Neither coracle nor actin is restricted to PSDs containing A-type receptors. Similarly, DLG is associated with a large area of the postsynaptic membrane rather than exclusively localized to B-type receptor clusters (Chen and Featherstone, 2005). Thus, data suggest that DLG, coracle, and actin are each necessary, but not sufficient, for glutamate receptor localization. How are receptors localized to discrete regions of the PSD? It is possible that spatially restricted subsets of postsynaptic DLG, coracle, and/or actin protein are functionally modified, and this functional modification might be required for interactions with glutamate receptors. However, what controls the spatial specificity of this functional modification? Ultimately, receptor localization is likely to be controlled by a transynaptic scaffold (such as neuroligin-neurexin) extending from presynaptic active zones to postsynaptic membranes. In the Drosophila NMJ, at least two transynaptic scaffolds would be required: one for localization of A-type receptors and one for localization of B-type receptors. Another parallel transynaptic scaffold might exist for postsynaptic localization of DLG, which clusters in the complete absence of postsynaptic glutamate receptors but not without presynaptic innervation (Chen and Featherstone, 2005).

In summary, we have shown that coracle interacts directly with Drosophila NMJ glutamate receptors in a subunit-specific manner and that coracle and actin are both required for anchoring of postsynaptic A-type but not B-type glutamate receptors. Our results are the first explanation for how Drosophila glutamate receptors might be anchored, and how A- and B-type receptors might be segregated from each other. Future work will determine how receptors are localized to specific regions of the postsynaptic membrane.

\section{References}

Aarts MM, Tymianski M (2004) Molecular mechanisms underlying specificity of excitotoxic signaling in neurons. Curr Mol Med 4:137-147.

Baines AJ, Keating L, Phillips GW, Scott C (2001) The postsynaptic spectrin/4.1 membrane protein "accumulation machine." Cell Mol Biol Lett 6:691-702.

Bellen HJ, Levis RW, Liao G, He Y, Carlson JW, Tsang G, Evans-Holm M, Hiesinger PR, Schulze KL, Rubin GM, Hoskins RA, Spradling AC (2004) The BDGP gene disruption project: single transposon insertions associated with $40 \%$ of Drosophila genes. Genetics 167:761-781.

Bolton MM, Blanpied TA, Ehlers MD (2000) Localization and stabilization of ionotropic glutamate receptors at synapses. Cell Mol Life Sci 57:1517-1525.

Chen K, Featherstone DE (2005) Discs-large (DLG) is clustered by presynaptic innervation and regulates postsynaptic glutamate receptor subunit composition in Drosophila. BMC Biol 3:1.

Coleman SK, Cai C, Mottershead DG, Haapalahti JP, Keinanen K (2003) Surface expression of GluR-D AMPA receptor is dependent on an interaction between its C-terminal domain and a 4.1 protein. J Neurosci 23:798-806.

De La Rue SA, Henley JM (2002) Proteins involved in the trafficking and functional synaptic expression of AMPA and KA receptors. ScientificWorldJournal 2:461-482.

DiAntonio A, Petersen SA, Heckmann M, Goodman CS (1999) Glutamate receptor expression regulates quantal size and quantal content at the Drosophila neuromuscular junction. J Neurosci 19:3023-3032.

Doussau F, Augustine GJ (2000) The actin cytoskeleton and neurotransmitter release: an overview. Biochimie 82:353-363.

Featherstone DE, Rushton EM, Hilderbrand-Chae M, Phillips AM, Jackson FR, Broadie K (2000) Presynaptic glutamic acid decarboxylase is re- 
quired for induction of the postsynaptic receptor field at a glutamatergic synapse. Neuron 27:71-84.

Featherstone DE, Davis WS, Dubreuil RR, Broadie K (2001) Drosophila $\alpha$ and $\beta$-spectrin mutations disrupt presynaptic neurotransmitter release. J Neurosci 21:4215-4224.

Featherstone DE, Rushton E, Broadie K (2002) Developmental regulation of glutamate receptor field size by nonvesicular glutamate release. Nat Neurosci 5:141-146.

Featherstone DE, Rushton E, Rohrbough J, Liebl F, Karr J, Sheng Q, Rodesch CK, Broadie K (2005) An essential Drosophila glutamate receptor subunit that functions in both central neuropil and neuromuscular junction. J Neurosci 25:3199-3208.

Fehon RG, Dawson IA, Artavanis-Tsakonas S (1994) A Drosophila homologue of membrane-skeleton protein 4.1 is associated with septate junctions and is encoded by the coracle gene. Development 120:545-557.

Kuromi H, Kidokoro Y (2003) Two synaptic vesicle pools, vesicle recruitment and replenishment of pools at the Drosophila neuromuscular junction. J Neurocytol 32:551-565.

Marrus SB, DiAntonio A (2004) Preferential localization of glutamate receptors opposite sites of high presynaptic release. Curr Biol 14:924-931.

Marrus SB, Portman SL, Allen MJ, Moffat KG, DiAntonio A (2004) Differential localization of glutamate receptor subunits at the Drosophila neuromuscular junction. J Neurosci 24:1406-1415.

Monaghan DT, Wenthold RJ, eds (1997) The ionotropic glutamate receptors. Totowa, NJ: Humana.

Nishikawa K, Kidokoro Y (1995) Junctional and extrajunctional glutamate receptor channels in Drosophila embryos and larvae. J Neurosci 15:7905-7915.

Ohara R, Yamakawa H, Nakayama M, Ohara O (2000) Type II brain 4.1 (4.1B/KIAA0987), a member of the protein 4.1 family, is localized to neuronal paranodes. Brain Res Mol Brain Res 85:41-52.
Parks AL, Cook KR, Belvin M, Dompe NA, Fawcett R, Huppert K, Tan LR, Winter CG, Bogart KP, Deal JE, Deal-Herr ME, Grant D, Marcinko M, Miyazaki WY, Robertson S, Shaw KJ, Tabios M, Vysotskaia V, Zhao L, Andrade RS, et al. (2004) Systematic generation of high-resolution deletion coverage of the Drosophila melanogaster genome. Nat Genet $36: 288-292$.

Petersen SA, Fetter RD, Noordermeer JN, Goodman CS, DiAntonio A (1997) Genetic analysis of glutamate receptors in Drosophila reveals a retrograde signal regulating presynaptic transmitter release. Neuron 19:1237-1248.

Qin G, Schwarz T, Kittel RJ, Schmid A, Rasse TM, Kappei D, Ponimaskin E, Heckmann M, Sigrist SJ (2005) Four different subunits are essential for expressing the synaptic glutamate receptor at neuromuscular junctions of Drosophila. J Neurosci 25:3209-3218.

Rosenmund C, Westbrook GL (1993) Calcium-induced actin depolymerization reduces NMDA channel activity. Neuron 10:805-814.

Scott C, Keating L, Bellamy M, Baines AJ (2001) Protein 4.1 in forebrain postsynaptic density preparations: enrichment of 4.1 gene products and detection of 4.1R binding proteins. Eur J Biochem 268:1084-1094.

Shen L, Liang F, Walensky LD, Huganir RL (2000) Regulation of AMPA receptor GluR1 subunit surface expression by a $4.1 \mathrm{~N}$-linked actin cytoskeletal association. J Neurosci 20:7932-7940.

Shirao T, Sekino Y (2001) Clustering and anchoring mechanisms of molecular constituents of postsynaptic scaffolds in dendritic spines. Neurosci Res 40:1-7.

Sprengel R, Aronoff R, Volkner M, Schmitt B, Mosbach R, Kuner T (2001) Glutamate receptor channel signatures. Trends Pharmacol Sci 22:7-10.

Ward REt, Lamb RS, Fehon RG (1998) A conserved functional domain of Drosophila coracle is required for localization at the septate junction and has membrane-organizing activity. J Cell Biol 140:1463-1473. 